Fecha de recepción: octubre 2021

Fecha de aprobación: noviembre 2021

Fecha publicación: diciembre 2021

\section{Relación moda-mujer en el siglo XVIII: prensa y representaciones}

Laura Pérez Hernández ${ }^{(1)}$

Resumen: La moda es un fenómeno social que transmite las particularidades de una época y del individuo que lo porta. A través de las modas se difunden diferentes identidades como la identidad colectiva o de género. La vinculación de la moda y su representación del género femenino ha estado presente en toda la historia. En este escrito se analizará el origen de la vinculación de las mujeres a estas concepciones de moda, representación, vanidad y comunicación en el siglo XVIII; cómo estas ideas recobran cada vez más fuerza en la prensa de esta centuria y de qué forma existe una continuidad de estas concepciones hasta la modernidad.

Palabras clave: moda - prensa - siglo XVIII - identidad - mujer - sociedad - historia - modernidad - género - representación.

[Resúmenes en inglés y portugués en la página 264]

(1) Licenciada en Historia, Facultad de Geografía e Historia, Universidad Complutense de Madrid, 2013. Máster en Historia de la Monarquía Hispánica, Facultad de Geografía e Historia, Universidad Complutense de Madrid, 2014. Doctorado en Historia y Arqueología, Facultad de Geografía e Historia, Universidad Complutense de Madrid. Doctor Mención Internacional, 2021. Becaria en Investigación Erasmus +, Spanish, Portuguese and Latin American Studies Department, University of Nottingham.

\title{
Introducción
}

La moda es un fenómeno social que transmite las particularidades de una época y de los individuos que lo portan ya sea su nivel social, estatus económico, identidad sexual, desarrollo cultural o etnia. El vestido, se entiende, pues como una forma de lenguaje que afecta al propio individuo, se convierte en un elemento simbólico que trata de representar algo. A través de las modas se difunden diferentes identidades como la colectiva o la de género. El vestido, además, refleja los patrones de conducta y formas de comportamiento sobre todo aquellos asociados al rango, el género y la clase social. Al principio de la historia, la ropa era tan solo una representación del poder. Tenía su rol principal en las Cortes de los 
Reyes o en los Imperios. El concepto de moda se utilizaba en un círculo muy concreto, en espacios donde los principales protagonistas eran viajeros, diplomáticos u hombres de negocio (Polan, 2006). Sin embargo, en el siglo XVIII, la moda se convierte en un tema de debate público. Con ella se discuten aspectos sobre el lujo, la belleza o la apariencia, y las primeras publicaciones periódicas que tratan esta cuestión en el siglo XVIII se convierten en el espejo donde las mujeres se miraban. Se repiten discursos que tratan de construir una imagen e identidad determinada para las mujeres de la época asociada a conceptos como el lujo, el consumo o la vanidad.

Esta vinculación de la vanidad y la moda con las mujeres ha estado presente en toda la historia de la humanidad. En el presente texto se pretende analizar el origen de estas concepciones, la relevancia que adquirieron en el siglo XVIII, como las ideas que relacionan a las mujeres con su interés en el consumo, el seguimiento de las tendencias y la renovación continua de las ropas cobra cada vez más fuerza en las publicaciones periódicas de la centuria, de qué forma se representan estas cuestiones en la prensa y cómo existe una continuidad de estas ideas en la transición del antiguo régimen al siglo XIX. Cuestiones que han continuado en épocas más modernas y que incluso se mantienen hasta la actualidad.

\section{Moda y Prensa en el Siglo XVIII: Estado de la Cuestión}

Hace dos décadas, Malcolm Barnard publicó su obra La moda como comunicación (Barnard, 2002) para debatir la idea de que las ropas tienen el rol de comunicar las identidades. El estudio de las publicaciones periodísticas de la moda o periodismo de moda en el que se analiza las formas en las que la moda se difunde en la historia es la clave para esto (Castaldo Lunden, 2020). Es precisamente en el siglo XVIII cuando la prensa empieza a actuar como difusora de la estética y apariencia femenina. Se trata de una cuestión poco explorada que nos ayudaría a comprender de una manera más compleja la moda como cultura y como industria (Castaldo Lunden, 2020). La época de la Ilustración permite explorar las intersecciones entre la moda y los medios de comunicación, como la prensa actúa como árbitro del gusto y de qué manera refleja las identidades tanto colectivas como de género. Los estudios de la historia de la moda fueron relegados a los ámbitos de la historia del arte y de la historia descriptiva hasta las últimas décadas del siglo XX. En los '90, aparecen las primeras aproximaciones al estudio de la moda desde nuevas perspectivas, como por ejemplo las relaciones entre los cambios en el traje y aspectos sociopolíticos en el caso de la obra de Ribeiro (Ribeiro, 1995) o la conexión entra la moda y el consumo en el caso de los estudios españoles hechos por Máximo García Fernández y Bartolomé Yun (García Fernández y Bartolomé Yun, 1997).

Sin embargo, no será hasta el siglo XXI cuando aspectos como el género, las identidades o las formas de comunicación de la moda salgan a la luz.

A nivel internacional destacan dos grandes autores, Guinard y Goffman. El primero (Guinard, 1976) analiza la importancia que adquiere la prensa en la Ilustración como forma de propaganda. De hecho, define a la prensa de moda como el fermento activo de la mentalidad de grupo. El segundo (Goffman, 1987) considera a la prensa como un medio para 
reflejar la identidad social, las interacciones y las expectativas de un mismo grupo. Una de las cuestiones en las que se centra es como la prensa establece algunos discursos sobre concepciones de los individuos, cuestiones de género o identidades que no se corresponden a la realidad social.

En el ámbito español, destacan estudios que vinculan el traje del siglo XVIII con las nuevas concepciones de feminidad (Bolufer, 1998), otros que analizan la relación existente entre la moda y la mujer a lo largo de la centuria (Cantos Fagoaga, 2007) e incluso algunos que utilizan los discursos de la prensa para analizar esta misma cuestión (Millet Viñes, 2001).

\section{La Vanidad y las Mujeres a lo largo de la Historia}

La relación que vincula a las mujeres con los conceptos de la moda, apariencia y vanidad ha estado presente en todas las épocas históricas. El uso que hacen las mujeres de las prendas para singularizarse y realzar su personalidad (Martínez Chacón, 1985) es una cuestión común a todas las épocas. Por ello, muchos de los principales teóricos de la moda (Martínez Chacón, 1985), han considerado este hecho como una de las razones de la relación establecida entre las mujeres y la vanidad (Simmel, 1961). Tanto la diferenciación sexual como la distinción social son indicadores que se expresan a través de la indumentaria, y estos, han ayudado al individuo a mostrarse de una forma diferente a los demás (Flügel, 1930). Como bien apuntaba Veblen en 1899, en su obra The Barbarian Status of Women (Veblen, 1899) las mujeres en su primer estadio económico se encuentran en un entorno hostil y se ocupan principalmente de las tareas relacionadas con la comida, la ropa y la apariencia de la casa (Veblen, 1899). Su principal dificultad es su dependencia del matrimonio, y, además, su función social está limitada por la coerción que ejerce su marido en ellas. Estas actividades que realiza le llevarán a desarrollar una serie de gustos y preferencias inclinadas, de acuerdo con Veblen, a la ornamentación.

Los primeros comentarios registrados sobre el atavío femenino aparecen en la época clásica, concretamente se discuten por primera vez en la obra de Tertuliano llamada Cultu Feminarum (Tertuliano, 2001). El discurso de la obra tiene como objetivo condenar el atavío de Eva y trata de difundir un modelo de mujer basado en la virtud y la decencia. Más tarde, la tradición eclesiástica utiliza esta vinculación de la mujer con la vanidad, la moda y apariencia para expresar su horror al sexo femenino (Bolufer, 2008).

Por supuesto esta concepción se mantiene durante el siglo XVIII, a través de los diferentes discursos literarios, sociales y periodísticos que apoyan y difunden esta cuestión convirtiéndola en un principio de la sociedad. Es, a partir de esta centuria, cuando la cuestión del binomio moda/mujer adquiere una mayor envergadura por diferentes razones empezando por la consolidación de la cultura de las apariencias que desde la centuria anterior se está desarrollando y siguiendo por la proliferación de textos periodísticos que debaten cuestiones sobre la apariencia. Si nos fijamos en el caso concreto de España, hay que tener en cuenta el gran interés que se muestra por los artículos y objetos que se introducen gracias a las modas internacionales. Entre ellos destacan todos aquellos productos, ornamentos y prendas que vienen de Francia. 


\section{Binomio Moda-Mujer en el Siglo XVIII}

En el siglo XVIII la imitación se convierte en una gran preocupación para los filósofos y teóricos sociales. De acuerdo con la teoría de la emulación, las modas empiezan en la cima de la escala social y se van filtrando en sentido descendente. Sin embargo, en el siglo XVIII y a medida que la sociedad moderna avanza este antiguo orden se rompe. La revolución del consumo provoca que grupos de clases medias, como los mercaderes, tengan acceso a nuevos productos que hasta ahora estaban destinados solo a las altas esferas sociales, rompiendo el orden del mundo (Entwistle, 2002). Las mujeres se convierten en las protagonistas de este consumo. En concreto, aquellas que se encarnan en un nuevo tipo de mujer, que ya participaba en los debates de la apariencia: las petimetras.

Las petimetras eran mujeres que estaban centradas en el deseo de consumir (Haidt, 2007), dirigiendo todas sus energías a la adquisición de productos y adornos. En especial están interesadas en los productos importados que les permitan estar a la última moda. En sus comportamientos atentan contra los modelos de feminidad establecidos, puesto que, rechazan el cumplimiento de sus obligaciones sociales y morales, dejando a un lado las relacionadas con su papel de esposas y madres. Anteponen a ellas la construcción de su apariencia y el cultivo de las modas y placeres (Haidt, 2007), al mismo tiempo que hacen gala de sus opiniones en los espacios de sociabilidad que van surgiendo. De ahí que su presencia dé lugar a debates centrados en evitar las nuevas invenciones de la moda por considerarlas culpables de todos los males sociales.

La vinculación de las mujeres de forma continua con el consumo y la vanidad obedece a diferentes razones: en primer lugar, porque siempre se ha considerado que la esencia femenina está en su materia y es totalmente contraria al espíritu. De acuerdo con algunos teóricos de la época, su esencia se refiere únicamente al cuerpo y a la apariencia (Tsëelon, 1995). De ahí que, sus inclinaciones siempre tenderán hacia lo frívolo y su comportamiento siempre estará lleno de peligrosidad. Por la naturaleza de la esencia femenina, es necesario tener controlada su sexualidad y apariencia constantemente. De acuerdo con la moral de la época: la mujer debe ser pura pero también parecerlo (Tsëelon, 1995).

Sin embargo, las concepciones sobre la esencia femenina no acaban aquí. También se les ha definido como el sexo desordenado por excelencia porque de acuerdo con la teoría de los humores, la mujer es más proclive a las enfermedades emocionales y la única manera de poder modificar su comportamiento es mediante la educación selectiva basada en los deberes morales (Davis, 1990).

En segundo lugar, existía una motivación de carácter social para esa vinculación entre el sexo femenino y la vanidad. De alguna manera necesitaban justificar el segundo plano asignado a las mujeres tanto en el ámbito social como en el público, y la idea de su inferioridad frente a los hombres junto con aquella que las relacionaba con lo frívolo y la apariencia con los aspectos banales de la vida, venían a justificar la diferente asignación de espacios según el género.

Un tercer grupo de motivaciones tiene carácter económico. La crisis económica del siglo XVII dejó devastada la economía española y supuso el fin del estilo español. De nuevo se necesitaba buscar responsables de la situación y de nuevo la inclinación de las mujeres al consumo apareció señalada entre las causas principales de la crisis económica. Ellas, 
eran consideradas las principales consumidoras de los nuevos productos extranjeros y las principales responsables del descenso de los matrimonios. Se les acusaba de tener comportamientos siempre inclinados a las novedades en las modas mientras sus vicios hacían que ningún hombre los quisiera para el matrimonio.

El exceso en los gastos debido a su excesivo consumo y el poco crecimiento de la natalidad por el poco empeño que ponían en procrear les aislaban de sus obligaciones sociales como esposas y madres (Martínez Chacón, 1985).

En último lugar, podemos considerar que esta relación de la mujer con la vanidad o la apariencia tuvo también motivos morales y estéticos. La mujer, educada en la modestia y la decencia desde prácticamente su nacimiento, tenía la necesidad de cubrir su cuerpo por pudor. Tal y como afirma Luján en su obra, existía una idea tradicional de vincular a las mujeres con las prendas por la necesidad que tenían de cubrir su cuerpo, así como de liberalizar algunas de sus partes (Luján, 1988).

Las principales críticas que sufren las mujeres a lo largo del siglo XVIII están sujetas a estas concepciones expuestas y se acentúan más por la presencia del nuevo tipo social de la petimetra, quien refleja un modelo de mujer totalmente vinculado a la vanidad y a la apariencia. Se dice que la petimetra genera las principales tensiones culturales debido a su excesivo consumo de productos internacionales en detrimento de fomentar el comercio nacional. Además, se considera que su comportamiento, sus inclinaciones y apariencias consiguen convertir lo francés en preferencia (Haidt, 2007).

\section{Difusión de Ideas sobre la Moda y las Mujeres en la Prensa del Siglo XVIII}

Estas ideas y concepciones que vinculan a las mujeres con el consumo, la vanidad y la moda se han difundido en el ideario colectivo de maneras muy diversas. Se recogen, por ejemplo, en la obra de Jovellanos sobre el origen del lujo. El autor, expone una paradoja:

El consumo del lujo no señala a los privilegiados de la sociedad sino a aquellos que tienen como único objetivo sobresalir mediante la estética, el vestido o el adorno. De acuerdo con los principios de la época, atribuye al sector femenino estos comportamientos. Considera que fomentan su desestima usando la ornamentación en exceso y cuando demuestran que su única preocupación es la apariencia exterior (Jovellanos, 1847, p. 70).

En sentido similar esta cuestión se manifiesta en la prensa de la época, en la que abundan las referencias hacia las formas de representación femenina con las diferentes indumentarias. Y no solo aparecen en los principales diarios, sino que también se mencionan en revistas misceláneas, poesías, artículos o crítica literaria (Bolufer, 1988).

La prensa en el siglo XVIII se convierte en un instrumento cultural que con el paso del tiempo alcanzará el nivel de foro de la opinión pública. Mediante ella se construyen las identidades de los individuos, ya que se dan descripciones sobre su aspecto, apariencia, vestimenta y hábitos. Los periódicos de la época no solo reflejan la estética de los diferen- 
tes grupos sociales, sino que además adquieren un valor intelectual siendo productos que merecía la pena comprar (Bartlett, Cole y Rocamora, 2013).

En las publicaciones periódicas, la moda se entiende como un índice de riqueza, privilegio social y estatus en el Antiguo Régimen (Mcllvanney, 2019) Jones en su trabajo Sexing la mode: Gender, Fashion and Commercial Culture in Old Regime Fashion (Jones, 2004) estudia el posible efecto que ejerció la moda en las mujeres del siglo XVIII. La moda era una parte de la élite social y jugó un rol importante en la creación de la ciudadanía y participación pública en una sociedad con un nuevo gusto (Jones, 2004).

Los escritores de las publicaciones periódicas del siglo XVIII han actuado como árbitros culturales y estéticos de la moda, clasifican las tendencias e innovaciones desde que aparecen estableciendo niveles de jerarquía entre aquellas importantes y otras más triviales (Best, 2017). Los diversos periódicos de la ilustración española tratan la cuestión de la estética a modo de debate y nos ayudan a reconstruir la vida tanto social como cultural de las mujeres de la época, así como sus comportamientos, modos de conducta y relación con el lujo. Prolifera la prensa femenina que define las conductas y comportamientos que deben seguir las mujeres. Estos discursos son una gran aportación a la cultura ilustrada porque están plagados de textos aleccionadores sobre cómo debería ser la educación, vida y comportamientos de las mujeres (Urzainqui Miqueleiz, 2016). Así, algunos autores, como Roig Castellanos la definen como el mejor medio de persuasión (Roig Castellanos, 1977). Entre los textos femeninos podemos destacar artículos, reseñas, comentarios al hilo de una noticia, cartas enviadas al periódico, etc. Documentos que ofrecen un gran caudal de reflexiones tanto sobre la mujer como consejos sobre cómo debería ser ésta y su particularidad es que no solamente están realizados por voces masculinas sino también que son las mismas mujeres las protagonistas (Capel Martínez, 2010).

Podemos ver algunos discursos donde se explica la relación que se establece entre la moda y la mujer en la prensa española de la época Ilustración a través de diferentes ejemplos.

Si hacemos un repaso cronológico de la prensa de finales del siglo XVIII nos encontramos con diversas descripciones de este modelo o tipo de mujer que solo le preocupa su apariencia y que encuentra su motivación en cambiar continuamente sus prendas.

El primer periódico que podemos mencionar es el diario catalán El Calaix de Sastre Catalán, del mismo se conservan dos ejemplares dedicados a la apariencia. En ellos se describe la vida de la petimetra tanto por el día como por la noche.

La petimetra, se dice, va ataviada desde el comienzo del día. Se despierta ataviada en su misma cama, donde viste varias prendas. Para la parte superior de su cuerpo utiliza una camisa, cubre sus piernas con las enaguas y además recoge su pelo con un accesorio denominado redecilla (Tarazona, 1761). Todos estos elementos de su apariencia los lleva desde el principio del día, desde que se despierta por lo que para la petimetra la apariencia e indumentaria tienen importancia desde la primera hora de la mañana. Una vez que la petimetra se ha levantado lo primero que hace es mirarse al espejo para comprobar su estado. Se enfada con él y vuelve a la cama maldiciendo su apariencia. El relato del día a día de la petimetra continúa, desde su habitación, sin ni siquiera haber comenzado el día ni realizado ninguna actividad, se queja de lo fatal que luce su apariencia. Con ella, en su habitación solamente se encuentra su criada, a la que muestra su miedo al espejo diciendo: Quita ya ese cristal que me da miedo al mirarme (Tarazona, 1761). 
En algunos periódicos de finales de siglo, El Correo de los Ciegos de Madrid de 1786, por ejemplo, se afirma que el matrimonio está en desuso por la mala educación que tienen las doncellas. Y se añade, que éstas suelen dedicarse en mayor medida a su apariencia exterior y hacen que los hombres no quieran casarse con ellas: “ ¿Cómo se han de animarse a casarse el hombre sensato y de medianas facultades viendo que las doncellas gastan el mismo o mayor tren que las rigurosas petimetras?" (Nipho, 1786, s/p).

En algunos otros ejemplos se culpa a las mujeres de la tiranía de la moda, es decir, de las continuas novedades que se desarrollan en los estilos que dominan el siglo XVIII. Se consideraba que eran las propias mujeres las que con su comportamiento inestable modificaban su indumentaria queriendo diferenciarse de las demás. Así, en uno de los grandes periódicos de la época publicado en 1781, El Censor, se dice que las petimetras que venían de las provincias a la corte tenían que quedarse un día sin salir y sin mostrarse a las gentes para evitar así ser la risa y el escarnio de cuantas la vieran (García Cañuelo y Heredia, 1781, s/p). Las continuas novedades en la moda no solo afectan a las provincias sino también a las mujeres de la propia ciudad de Madrid en el caso de que se ausentaran. De acuerdo con El Censor, si las madrileñas se ausentaban un tiempo, aunque fuera breve necesitaban unos días para ponerse al día en las modas. En uno de sus ejemplares, el citado periódico pone un ejemplo de lo que ocurrió con una mujer que estuvo fuera durante seis meses:

Sé de una que estuvo fuera como cosa de unos seis meses, y a la vuelta fue preciso rociar, y dar algunas fumigaciones a sus hijas que creyeron que era su abuela, que venía a visitarles desde el otro mundo, se accidentaron del susto. Tanta era la antigüedad del traje que traía (García Cañuelo y Heredia, 1781, s/p).

Por último, aparecen críticas contra la nueva vida que tienen las mujeres y su obsesión por las novedades en la moda. De acuerdo con estos comentarios, una de las principales razones por las que las mujeres no quieren tener o criar a sus hijos es porque quieren mantener el aire y el talle de petimetra.

De acuerdo con El Pensador (Clavijo y Fajardo, 1765), las petimetras no pueden dejar en ningún momento de utilizar la cotilla y por ello descienden en tanta medida los nacimientos. Estas mujeres no quieren amamantar a sus hijos porque están más preocupadas por las longitudes de sus cuerpos. Las modas hacen que las madres se pasen horas en el tocador y gasten el tiempo en peinar sus rizos, que lo único que les provoca eran dolores de cabeza. También debido a la moda se desarrollan muchas más enfermedades porque algunas prendas como la cotilla oprime de tal manera sus estómagos que apenas les permite que su cuerpo se mueva de forma natural. Lo mismo ocurre con otras prendas y complementos como los cuellos que les aprietan o algunos de los zapatos definidos como "grilletes que apenas les permiten dar un paso" (Clavijo y Fajardo, 1765, p. 25).

No sabemos si este tipo de mujer descrita en los artículos periodísticos es real o responde a un estereotipo creado por el discurso social con el fin de mantener la relación de la mujer con la vanidad y la estética y así poder justificar su inferioridad. Todos los discursos sociales que hemos visto hasta ahora consideran el consumo femenino como la principal raíz del mal (Sarasúa, 1996). Sin embargo, existen otras opiniones que se oponen a la concepción que relaciona lo femenino con la frivolidad y la estética. Este es el caso de Feijoo, en 
1764 publica su obra El Teatro Crítico Universal (Feijoo, 1764) defendiendo a las mujeres. Es cierto que considera que la moda puede arrastrar a las mujeres a los vicios y las incita a una continua preocupación por la belleza e innovación de sus prendas (Feijoo, 1764). Sin embargo, para el autor el mayor problema se da en su entendimiento. Es cierto que las mujeres caen en los vicios y son perversas, pero esto también les ocurre a los hombres. Afirma que en el siglo XVIII defender a las mujeres era lo mismo que ofender a los hombres. No se admite nada bueno de ellas ya que en lo moral se les llena de defectos mientras que en lo físico se les considera que están plagadas de imperfecciones: "No es ya solo vulgo ignorante con en el que entró en la contienda: defender a todas mujeres viene a ser lo mismo que ofender a casi todos los hombres" (Feijoo, 1764, p. 377). En sus textos rechaza la concepción clásica que vincula a las mujeres con los vicios empezando por desmentir las particularidades negativas que les atribuía a las mujeres desde el pasaje de Adán y Eva. Feijoo considera que si bien fue Eva quien incitó a Adán al pecado, también fue un ángel quien incitó a ésta a cometer el pecado. Por lo tanto, no se puede probar la maldad de la mujer mediante este episodio. Se aleja de esta concepción femenina que asocia a la mujer con la vanidad poniendo otros ejemplos de mujeres célebres como Catalina de Médicis u otras mujeres que habían gobernado de una forma excelente.

Para él, las mujeres no pueden ser la causa de los grandes gastos porque existen muchos ejemplos de grandes mujeres que han gestionado la economía doméstica. De acuerdo con la obra de Feijoo, el poco entendimiento femenino en materias que va más allá de lo doméstico, la estética o la ornamentación no se debe a la falta de sus capacidades para desarrollarse, sino a que no han tenido nunca acceso a la instrucción para poder desarrollar su inteligencia (Feijoo, 1764). Se opone totalmente a la teoría de que las mujeres son frívolas y cuestiona la concepción de inferioridad femenina. Por ejemplo, en uno de sus pasajes habla de una mujer que tiene un gran conocimiento en cuestiones domésticas, en las que centra todo su pensamiento. Sin embargo, su marido de inferior talento es capaz de controlar las materias de esfera superior por el hecho de frecuentar relaciones con gente de afuera, como religiosos, sabios y hábiles políticos. De ellos, recibe noticias sobre los negocios, advertencias, informaciones sobre cuestiones políticas, etc. De esta manera su marido está instruido en varias materias y si, en alguna ocasión, trata éstas delante de su mujer y ella le dice algo que se le ocurre, por muy penetrante que sea no puede ser a la altura del conocimiento de su marido. Y esto se debe a que, en este caso, esta mujer está desnuda de toda instrucción y aunque esté en lo cierto nunca tendrá mayores informaciones que su marido. Este es el motivo por el que ante ciertas materias el marido queda como un lince y ella como una tonta; sin embargo, no es una cuestión basada en el entendimiento de cada uno o en la falta de talento femenino, sino en su falta de noticias. En su obra expresa que no pretende hacer una defensa de superioridad de las mujeres sino de igualdad entre ambos y lo expresa de la siguiente manera: "Mi empeño no es persuadir la ventaja de las mujeres frente a los hombres sino la igualdad" (Feijoo, 1764, p. 380).

Más allá de la defensa de las mujeres de Feijoo, encontramos algunas reivindicaciones en la prensa por parte de las voces femeninas, es el caso de las pensadoras Pensatriz Salmantina y Pensadora Gaditana. A través de sus artículos describen la acción de las mujeres en diferentes espacios, considerando que, aunque consuman gran parte de su tiempo en el tocador, el escritorio es uno de los lugares que también deben frecuentar. Sin embargo, 
la Pensatriz considera que la defensa de las mujeres es muy difícil de difundir en esta sociedad puesto que hay muchos testimonios que la señalan como un individuo inferior y sin raciocinio. Pone el ejemplo de los textos religiosos, siguiendo las Sagradas Escrituras mediante las que se establece que las mujeres no tienen alma racional: La Pensatriz dice parafraseando a las escrituras: "Las mujeres no tenemos alma racional ni somos de la especie humana” (Hurtado Girón y Silva de Pico, 1777, p. 4).

La Pensatriz Salmantina en uno de sus ejemplares crítica a los hombres al ser los que establecen cómo deben ser las apariencias femeninas. Además, hace intentos de demostrar la racionalidad de las mujeres construyendo un listado de aquellas mujeres que han tenido cierta posición o relevancia en la sociedad y se centra en las que han aportado un conocimiento científico o filosófico importante. Explica las vidas de mujeres como Juana Morella o Inés de la Cruz. Pide que no se reduzca el conocimiento de la mujer a una sola cuestión y lo refleja considerando que no se puede reducir toda ciencia femenina y toda comprensión de la mujer a la aguja. Defiende la capacidad de las mujeres para usar la razón y desarrollarse en materias científicas y filosóficas (Hurtado Girón y Silva de Pico, 1777).

Tanto Feijoo como la Pensatriz Salmantina están señalando que las mujeres tenían suficiente presencia como para que fuera cuestionable su no participación en la nación y su continua relación con los ámbitos más frívolos de la sociedad como la moda o la estética.

\section{Conclusiones}

En la actualidad los medios de comunicación construyen una imagen específica de las mujeres, son los espejos en los que ellas se miran para alcanzar un ideal concreto. Como hemos visto, a lo largo del artículo, el origen de la difusión de estas ideas asociadas a las mujeres parte de la prensa del siglo XVIII. Sin embargo, la conexión establecida entre las mujeres y la vanidad, o entre el género femenino y su interés por la estética comienza mucho antes en la época clásica. A partir de ahí se construye todo un entramado formado por la tradición eclesiástica y la objetividad masculina, en que las mujeres son siempre las protagonistas en el consumo, las culpables de la ruina de las naciones y las que cometen mayores excesos con sus ropas.

El siglo XVIII resulta una fecha clave para empezar a analizar estas cuestiones ya que es en este momento cuando los periódicos se convierten en los medios de difusión de las maneras y comportamientos, siendo un elemento fundamental de la cultura de la época. Los artículos que en ellos se presentan describen la vida cotidiana de diferentes tipos sociales como es el ejemplo utilizado de la petimetra, pero también abordan cuestiones más profundas y plantean debates sobre temas complejos como la belleza, el lujo o la moda. A través de estos debates y ensayos se construyen discursos relativos a la sociedad, hay un claro rechazo a la introducción de novedades en las modas por ser las culpables de corromper los comportamientos femeninos. Estos debates también construyen un discurso político proponiendo la naturalización de las modas y los comportamientos que se han de seguir por parte del género femenino. Por último, se construye también un discurso de género, Algunos debates que se plantean dan pistas de cómo se construían las identidades feme- 
ninas. Se producen continuas críticas a las mujeres por tener cierta tendencia al consumo y se propone un modelo de comportamiento más comedido, así como un vestido que no dé lugar a excesos. Aparece un discurso que vincula a la mujer con la vanidad y considera está inclinación algo que está en su propia naturaleza. Este discurso aparece con frecuencia en la mayoría de los periódicos que actúan como críticos sociales y tienen voz masculina. Se define a las mujeres como la causa de la ruina de las naciones y se señala su comportamiento artificial y tendencia a los nuevos productos como la razón de decrecimiento de los matrimonios y la falta de moral en la sociedad de la época.

Las continuas críticas a las mujeres y su relación con las ruinas económicas crean algunas crispaciones como las señaladas en la obra de Feijoo. Se cuestiona por primera vez en la historia la inferioridad de las mujeres en el ámbito intelectual, se considera que su falta de formación no se debe a su menor inteligencia sino a que han tenido menos oportunidades para acceder a la educación. Surgen además voces femeninas que se quejan ante esta situación, destacan las principales pensadoras como la mencionada pensadora gaditana $o$ la pensatriz salmantina. Podemos considerar, el siglo XVIII como el primer momento en el que se cuestiona la situación de las mujeres. Desde esos años, el problema adquiere un nivel público y se convierte en tema de debate social. Sin embargo, las expectativas tan altas a la hora de cambiar las ideas sobre la naturaleza y capacidades de las mujeres mayoritariamente aceptadas quedarían insatisfechas. Es verdad que las mujeres se convirtieron en las protagonistas de los debates periodísticos y empezaron a quejarse contra los principios establecidos, pero siguen siendo señaladas culpables de los males de la sociedad, como lo prueba la continua relación que se hace de ellas con los abusos y males a los que conduce el lujo. Estas concepciones se mantienen más allá del Antiguo Régimen, en el siglo XIX la prensa de moda jugó un papel fundamental en convertir a las prendas y su consumo como un elemento central de la identidad femenina (Velasco Molperecres, 2015), revistas europeas como la Belle Aseemblée, Lady's Magazine o the World of Fashion fueron parte del entretenimiento de las mujeres de la época en ellas se promueven las virtudes domésticas, las buenas maneras y los artículos de moda (Polan Brenda, 2006). La conexión entre la feminidad y el consumo traspasa el siglo XVIII siendo protagonistas en épocas más modernas e incluso llegando hasta la actualidad.

\section{Bibliografía}

Barnard, M. (2002). Fashion as Communication, London: Psychology Press.

Bartlett, D. Cole, S. y Rocamora, A. (2013). Fashion Media: Past and Present. London: Bloomsbury Academy.

Bolufer, M. (1998). La construcción de la feminidad en la España del siglo XVIII, Valencia: Institució Alfons El Magnanim.

Bolufer, M. (2008). Mujeres y modernización: estrategias culturales y prácticas sociales (Siglos XVIII-XX), Madrid: Instituto de la Mujer.

Cantos Fagoaga, M. (2007). La indumentaria: indicador económico y sociocultural. Torrent Siglo XVIII, Estudis (33), 287-298. 
Capel Martínez, R. Ma , (2010). Prensa y Escritura femenina en la España Ilustrada, El Argonauta Español (7).

Castaldo Lundén, E. (2020). Exploring Fashion as Communication: The Search for a new fashion history against the grain, The International Journal of Media and Culture, (4), 249-258.

Clavijo y Fajardo, J. (1762). El Pensador, Madrid: Imprenta de Joachin Ibarra.

Davis, N. (1990). Un mundo al revés en Historia y género: Las mujeres en la Europa moderna y contemporánea, por J. Amelang y M. Nash. Valencia: Alfons el Magnanim.

Enwistle, J. (2002). El cuerpo y la moda: una visión sociológica, Barcelona: Paidós.

Garcia Fernandez, M. y Yun, B. (1997). Pautas de consumo: estilos de vida y cambio político en las ciudades castellanas a fines del Antiguo Régimen, en Imágenes de la diversidad. El mundo urbano en la Corona de Castilla: siglos XVI-XVIII, por J.I Fortea Perez (coord.). Santander: Universidad de Cantabria.

García Cañuelo y Heredia, L. (1781). El Censor, Madrid.

Feijoo, B. (1734). Teatro Critico Universal ó discursos varios en todo género de materias para desengaños de errores comunes, Pamplona: Benito Coscuella.

Flugel, G. (1930). The psychology of clothes, The International Psycho-analytical Library (18).

Goffman, E. (1987). La representación de la vida cotidiana, Madrid: Ed. Martínez de Bugía.

Guinard, P. (1976). La presse espagnole de 1737 à 1791 formation et signification d'un genre, Paris: Centre de Recherches Hispaniques, Institut d’Études Hispaniques.

Haidt, R. (2007). Emoción o aplicación Petimetra y la economía del deseo, Cuadernos de Ilustración y Romanticismo (15), 155-173.

Hurtado Giron y Silva de Pico, E. (1777). La pensatriz salmantina, Salamanca: Oficina de Sta. Cruz por Domingo Casero.

Jones, J. Sexing la mode: Gender, Fashion and Commercial Culture in Old Regime Fashion, London: Berg.

Jovellanos, G. M. (1847). El origen de lujo, Obras de Don Gaspar Jovellanos Nueva Edición, Tomo VII, Logroño: Imprenta de Don Domingo Ruiz.

Lujan, N. (1988). La vida cotidiana en el siglo de oro español, Barcelona: Planeta.

Mcllivanney (2019). Figurations of the feminine in the Early French Women's Press 1758-1848, Liverpool: University Press.

Millet Vines, C. (2001). La difusión de la moda a través de las publicaciones periódicas, II Jornadas Internacionales sobre moda y sociedad, Granada: Universidad de Granada.

Nelson Best, K. (2017). The History of Fashion Journalism, London: Bloomsbury.

Nipho, M. (1786). El Correo de los Ciegos de Madrid, Madrid.

Polan, B. (2006). Fashion Journalism in The Fashion Handbook by T. Jackson and D. Shaw, London: Routledge.

Ribeiro, A. (1995). The art of dress: Fashion in England and France 1750-1820, New Haven and London: University Press.

Roig Castellanos, M. (1977). La mujer y la prensa: desde el siglo XVIII a nuestros días, Madrid: La autora.

Simmel, G. (1961) La cultura femenina y otros ensayos, México: colección Austral.

Tarazona, P.A. (1761). El caxón de Sastre Cathalan, Barcelona: Imprenta de la Gaceta.

Tertuliano, A. (2001). De cultu Feminarum. El adorno de las mujeres, Málaga: Servicios de Publicaciones de la Universidad de Málaga. 
Tsëelon, E. (1995). The masque of femininity: the presentation of woman in everyday life, London: Sage Publications.

Urzainqui Miqueleiz, I. (2016). La prensa española como modeladora de la conducta femenina en M. García Hurtado El siglo XVIII en femenino: las mujeres en el Siglo de Las Luces, Madrid: Síntesis.

Veblen, T. (1899). The Barbarian status of women, American Journal of Sociology, (4), 503-514.

Velasco Molpeceres, A.M. (2015). Moda y prensa femenina en España (Siglo XIX), Madrid: Ediciones 19.

Abstract: Fashion is a social phenomenon that transmits the particularities of an era and the individual who wears it. Through fashions, different identities such as collective or gender identity are disseminated. The linking of fashion and its representation of the female gender has been present throughout history. In this writing the origin of the connection of women to these conceptions of fashion, representation, vanity and communication in the 18th century will be analyzed; how these ideas regain more and more force in the press of this century and in what way there is a continuity of these conceptions until modernity.

Keywords: fashion - press - 18th century - identity - woman - society - history - modernity - gender - representation.

Resumo: A moda é um fenômeno social que transmite as particularidades de uma época e de quem a veste. Por meio da moda, diferentes identidades, como identidade coletiva ou de gênero, são disseminadas. A vinculação da moda e sua representação do gênero feminino esteve presente ao longo da história. Neste escrito, será analisada a origem da ligação das mulheres a essas concepções de moda, representação, vaidade e comunicação no século XVIII; como essas ideias ganham cada vez mais força na imprensa deste século e de que forma há uma continuidade dessas concepções até a modernidade.

Palavras chave: moda - imprensa - século 18 - identidade - mulher - sociedade - história - modernidade - gênero - representação.

[Las traducciones de los abstracts fueron supervisadas por el autor de cada artículo] 